\title{
Mitophagy as a stress response in mammalian cells and in respiring $S$. cerevisiae
}

\author{
Hagai Abeliovich*† and Jörn Dengjelł $\S \|$ \\ "Department of Biochemistry and Food Science, Hebrew University of Jerusalem, Rehovot 76100, Israel \\ †Freiburg Institute for Advanced Studies (FRIAS), University of Freiburg, Albertstr. 19, 79104 Freiburg, Germany \\ \$Department of Dermatology, Medical Center, Freiburg Institute for Advanced Studies (FRIAS), University of Freiburg, Albertstr. 19, 79104 Freiburg, Germany \\ §ZBSA Center for Biological Systems Analysis, University of Freiburg, Habsburgerstr. 49, 79104 Freiburg, Germany \\ \|Department of Biology, University of Fribourg, Chemin du Musée 10, 1700 Fribourg, Switzerland
}

\begin{abstract}
The degradation of malfunctioning or superfluous mitochondria in the lysosome/vacuole is an important housekeeping function in respiring eukaryotic cells. This clearance is thought to occur by a specific form of autophagic degradation called mitophagy, and plays a role in physiological homoeostasis as well as in the progression of late-onset diseases. Although the mechanism of bulk degradation by macroautophagy is relatively well established, the selective autophagic degradation of mitochondria has only recently begun to receive significant attention. In this mini-review, we introduce mitophagy as a form of mitochondrial quality control and proceed to provide specific examples from yeast and mammalian systems. We then discuss the relationship of mitophagy to mitochondrial stress, and provide a broad mechanistic overview of the process with an emphasis on evolutionarily conserved pathways.
\end{abstract}

\begin{abstract}
Mitochondria, genetic disease and aging
Mitochondria perform a variety of essential physiological functions in all eukaryotic cells. The production of ATP by oxidative phosphorylation is clearly the best publicized of these roles. However, no less essential are the roles played by mitochondria in fatty acid oxidation and the biosynthesis of isoprenoids, Haeme, amino acids, iron-sulfur clusters and nucleotides, to mention a few. In fact, the capability of mitochondria to perform oxidative phosphorylation comes at a price. Breakdown of the chemical potential capacitor function of the mitochondrial inner membrane leads to nonproductive partial reduction in molecular oxygen to form reactive oxygen species (ROS) [1]. Although ROS affect the cell as a whole, mitochondria suffer a disproportionate level of oxidative damage due to proximity effects. This damage is manifested in mutagenesis of mtDNA, as well as in oxidation reactions such as protein glycation and lipid oxidation. In addition, disruption of mitochondrial compartmentalization results in leakage of cytochrome $\mathrm{c}$ and other cytotoxic factors. Mitochondria with defective chemiosmotic coupling can 'short circuit' the process, leading to an energy drain. Gradual accumulation of mtDNA mutations has been invoked as an explanation for aging phenomena. In addition, many maternally-inherited forms of late onset diseases, such as type II diabetes, deafness, mitochondrial encephalomyopathy, and optic neuropathy, to mention a few, have been linked to maternally-inherited mutations in the mitochondrial genome
\end{abstract}

Key words: autophagy, degradation, mitochondria, stress response. Abbreviations: IPSC, induced pluripotent stem cell; PD, Parkinson's disease; SILAC, stable isotope labelling by amino acids in cell culture.

"To whom correspondence should be addressed (email ahagai@agri.huji.ac.il).
$[2,3]$. These findings have led to a unified 'mitochondrial theory of aging'. Within the framework of this theory, a role has been proposed for a quality control mechanism that identifies malfunctioning mitochondria, and prevents clonal takeover of the system by defective mitochondrial cohorts [2-5]. One such disease that is related to mitochondrial dysfunction is Parkinson's disease (PD). PD is a disorder in which neurons accumulate an amyloid form of $\alpha$ synuclein, a small, synaptic vesicle-associated protein [6]. This accumulation correlates with degeneration and death of dopaminergic neurons in the substantia nigra region of the brain, ultimately leading to major symptoms of the disease, such as resting tremor and rigidity of limbs. Agents which compromise mitochondrial function, such as rotenone and paraquat, induce a PD-like condition in rats [7]. Familial forms of PD are known to be caused by a set of mutations which map to specific genes, including those encoding the proteins $\alpha$-synuclein, LRRK2, DJ-1, ATP13A2, PINK1 and Parkin [8]. As discussed below, two of these proteins, PINK1 and Parkin, are directly involved in mitochondrial quality control and in mitophagy.

\section{Mitophagy as a quality control mechanism for culling defective mitochondria in mammalian cells}

Although mitochondria sport a wide spectrum of quality control mechanisms including endogenous proteases and proteasome-mediated degradation, the only known cellular mechanism that can actually mediate the degradation of entire organelles without spillage is, by definition, an autophagic mechanism. The first studies of selective mitochondrial 
autophagy came from the Lemasters lab (which also coined the term 'mitophagy'; [9-11]). Later studies confirmed the hypothesis that malfunctioning mitochondria are selectively degraded by mitophagy in mammalian cells $[12,13]$. It was reported that (1) depolarized mitochondria are unable to fuse with other mitochondria; (2) $85 \%$ of fission events lead to formation of one hyperpolarized compartment and one depolarized compartment, suggesting that an active process segregates intra-mitochondrial material; (3) in the mitochondrial life-cycle, the post-fission state is the 'resting state' and that fusion was rapidly followed by fission and finally (4) that depolarized mitochondria, formed by selective fission, are selectively autophagocytosed.

In mammalian cells, many mechanistic details of these steps have since been fleshed out. Several labs have shown that PINK1, a membrane anchored mitochondrial protein kinase [12,14-16], phosphorylates and activates Parkin, a ubiquitin-conjugating E3-type enzyme [17-19] in addition to phosphorylating ubiquitin itself [18-21]. The combination of phospho-Parkin and phospho-Ub functions as a feed-forward loop to amplify the signal [22]. Two phosphoubiquitin-specific autophagy receptors, optineurin and NDP52, have been shown to be recruited to Pink1-presenting mitochondria [23,24]. Interestingly, these same proteins were previously implicated in xenophagy $[25,26]$. Alternatively, it has been suggested that PINK1 phosphorylates mitofusin 2, and that this species functions as a Parkin receptor [27]. In parallel, Parkin directs the ubiquitination of $\mathrm{Mfn} 1$ and $\mathrm{Mfn} 2$, mitofusins that are required for mitochondrial fusion [28] as well as numerous other mitochondrial outer membrane proteins. Studies show that PINK1 is recruited to all mitochondria, but is degraded in an ubiquitin- and proteasome-dependent manner in energized mitochondria [16]. Although these results provide a mechanistic explanation for much of the data, several important questions remain. For example, as $85 \%$ of fission events lead to segregation between depolarized and hyperpolarized mitochondria [13], active segregation of functional and defective components must be taking place. Is this truly the case, how is this segregation linked to the PINK1 load of the outer mitochondrial membrane?

\section{Mitophagy in S. cerevisioe}

The initial report on autophagy in Saccharomyces cerevisiae [29] demonstrated, by EM, the presence of mitochondria in nitrogen-starvation-induced autophagic bodies, as was previously shown three decades earlier for mammalian cells [30]. These pictures, however, appear to be anecdotal: very few mitochondria are actually engulfed by autophagosomes during nitrogen-starvation of $S$. cerevisiae. In fact, despite numerous attempts, the standard nitrogen-starvation protocols (transfer from glucose-based minimal medium to glucose-based minimal medium lacking nitrogen; inhibition of TORC1 in minimal and rich glucose-based medium) which are used to assay standard macroautophagy in yeast, do not yield any discernable biochemical or structural manifestations of mitophagy.
The first report of general mitophagy occurring enmass in $S$. cerevisiae (as opposed to anecdotal EM pictures) came from studies of Kissova et al. [31]. They showed that yeast cells that are grown in rich lactate medium and transferred to minimal nitrogen-starvation on glucose, display mitophagy (as measured by transfer of a mitochondrially targeted GFP to the vacuolar lumen) within $12 \mathrm{~h}$. However, the procedure used is unlikely to reflect a quality control event since it involves a direct response to starvation coupled with a change in carbon source and additional unidentified media components.

Tal et al. [32] first showed that without outside intervention, wild-type yeast cells incubated until stationary phase under respiratory conditions (using lactate as carbon source) display massive mitophagy at very long time points, beyond 3 days of incubation. Several observations suggest that this phenomenon may represent a quality control process. Firstly, standard nitrogen-starvation protocols do not lead to mitophagy. In addition, it can be demonstrated that macroautophagy occurs already on day 1 of the 3-day stationary phase mitophagy protocol [33]. Hence, mitophagy is temporally distinct from starvation-induced macroautophagy. Furthermore, the genetic requirements for stationary phase mitophagy and for starvation-induced mitophagy (simultaneous change in carbon source and nitrogen availability) do not fully overlap [34].

Two groups, the Klionsky lab and the Ohsumi lab, took advantage of the stationary phase mitophagy protocol to carry out large-scale screening of the yeast knockout collection for strains that lost the ability to transfer mitochondrial GFP to the vacuole under these conditions. These screens identified a battery of interesting genes [3436]. The general message of these studies indicates that mitophagy is a type of selective autophagy. As in all other instances of selective autophagy, mitophagy requires most of the general autophagy machinery (for review, see [37]), and in addition to these, requires a specific mitophagy receptor. In the case of yeast mitophagy, this receptor was identified as Atg32, a type II integral membrane protein of the outer mitochondrial membrane (see Figure 1). Atg32 functions as a mitophagy receptor that interacts with components of the autophagy machinery, such as Atg11, that are specific to selective autophagic pathways such as the Cvt pathway and pexophagy, the selective degradation of peroxisomes. Although Atg32 seems to be expressed uniformly on yeast mitochondria, its overall levels are increased under mitophagy conditions in a redox-dependent fashion [36], and its overexpression causes an increase in the degree of mitophagy. In addition, it undergoes posttranslational modifications which may regulate mitophagy. It is phosphorylated on serine 114 in its N-terminal cytosolic domain [38], and it is proteolytically cleaved within its intermembrane space C-terminal domain by the AAA protease Yme1 [39]. The levels of Atg32 respond to the availability of glutathione [36], and glutathione levels can be manipulated by defects in phospholipid metabolism [40], although it is unclear to what degree changes in phospholipid metabolism accompany 
Figure 1 C Comparison of sequestering membrane recruitment to mitochondria in (A) yeast and (B) mammalian cells

In yeast $(\mathbf{A})$ activation of Atg32 requires a proteolytic clipping step, mediated by the AAA protease Yme1, as well as phosphorylation. Activated Atg32 is then able to interact with lipidated Atg8 as well as with the Atg11 scaffold protein. (B) In mammalian cells, loss of mitochondrial membrane potential stalls PINK1 import and stabilizes it on the outer membrane, by preventing constitutive PARL-dependent proteolysis of imported PINK1 (not depicted). The stabilized PINK1 phosphorylates both Parkin, as well as ubiquitin. This simultaneously recruits Parkin to mitochondria and activates it to ubiquitylate mitochondrial outer membrane proteins, which in turn are recognized by the redundant mitophagy receptors NDP52 and Optineurin, thus linking LC3-II (the mammalian orthologue of lipidated Atg8) with outer mitochondrial proteins.
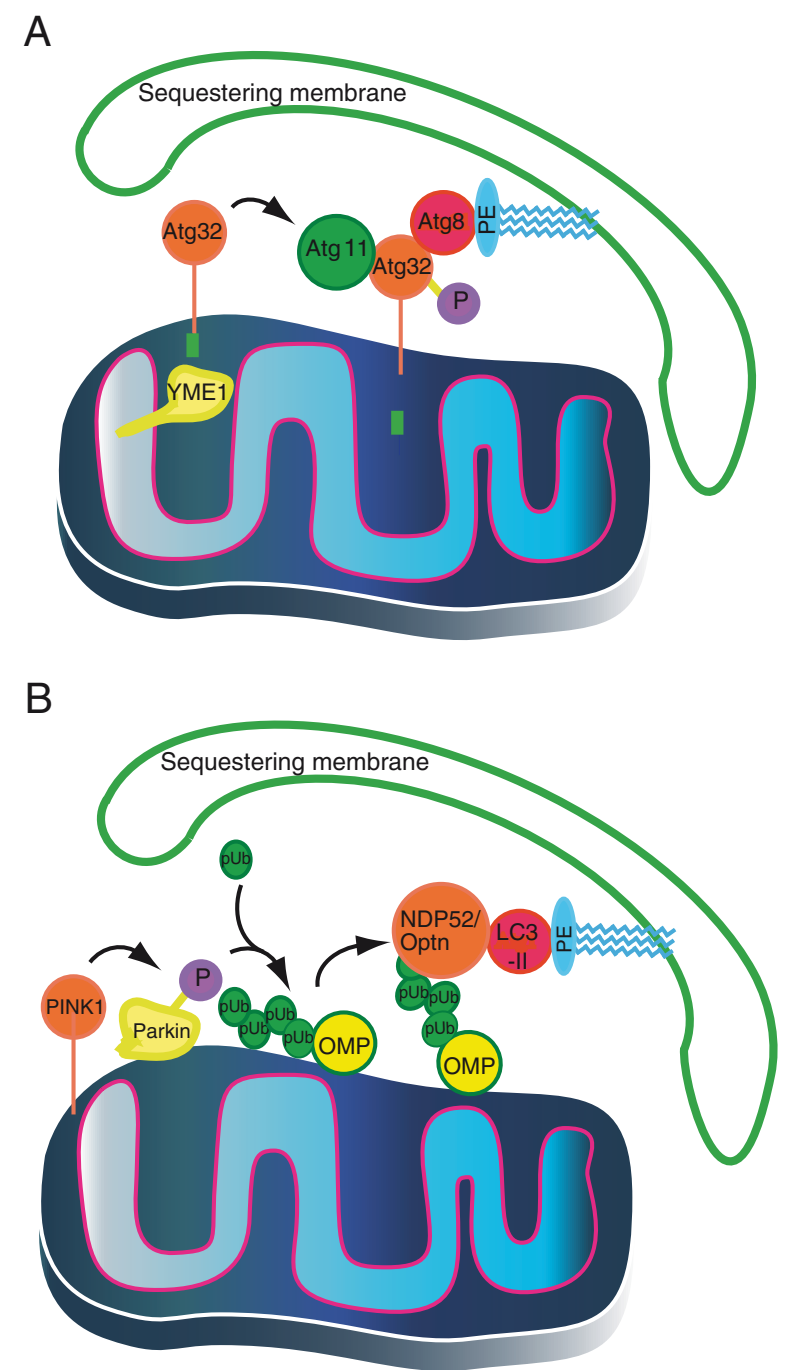

the onset of mitophagy in wild type cells. Although no direct orthologue of Atg32 is known in mammalian cells, it is clearly functionally analogous to mammalian mitophagy receptors such as optineurin and NDP52 (Figure 1).

Although additional mitophagy-specific proteins were identified through genetic screening in S. cerevisiae, current knowledge of their mechanistic involvement in mitophagy is rather preliminary at this point. Given the brief nature of this mini-review we refer the reader to the specific literature for further information [34-36].

\section{Redox stress, mitophagy and mitochondrial homoeostasis}

In the context of the mitochondrial theory of aging, one would like to be able to demonstrate that mitophagy plays a role in alleviating a potentially dangerous situation for eukaryotic cells. Hypothetically, nonfunctional mitochondria could either hamper the cell by being a metabolic burden, or by generating harmful substances such as reactive oxygen species (ROS). Paradoxically, some studies found no increases in the levels of ROS under conditions where mitophagy was blocked [35]. However, Twig et al. found an increase in oxidized proteins in COS7 cells expressing FIS1 RNAi or a dominant negative DRP1 mutant leading to mitophagy impairment. This result is corroborated in yeast by Journo et al., who found that aup $1 \otimes$ cells, which exhibit decreased mitophagy [32], accumulate more oxidized proteins than wild-type under stationary phase mitophagy conditions. Perhaps the most interesting results come from a study by Deffleu et al. They showed that the antioxidant N-acetylL-cysteine (NAC) can inhibit mitophagy in yeast, but not general nitrogen-starvation-induced macroautophagy. The effect on mitophagy was not due to changes in ROS levels, but to an increase in glutathione levels. Indeed, mutants in glutathione synthetase show a significantly higher level of mitophagy that is not inhibited by NAC. These results may suggest that accumulation of oxidized molecular species (possibly proteins) in the cell may trigger mitophagy, and also relates to the fact that NAC inhibits the expression of ATG32 [36].

\section{Aspects of mitophagy: targeting, segregation and engulfment}

Not all mitochondria are degraded during the induction of mitophagy in S. cerevisiae. At the very least, this is due to the fact that mitochondria are essential for cell survival. In addition, however, the data of Twig et al. [13] as well as Narendra et al. [16] suggest that depolarized mitochondria are generated specifically during fission events, and that these depolarized units are specifically targeted for mitophagy. Again, the fact that $85 \%$ of mitochondrial fission events were shown to result in one significantly depolarized and one significantly hyperpolarized daughter must imply that a non-random segregation process is taking place. Such segregation, coupled with fission, will ultimately 'distill' defective mitochondria out of the dynamic network. Combined with the conclusions of Whitworth and colleagues $[41,42]$, who showed that depolarization of mitochondria leads to ubiquitination of mitofusins, one can hypothesize that once segregation and fission have taken place, a dedicated mechanism generates a signature on the depolarized mitochondrion in order to identify the compartment as 
defective. In mammalian cells, this signal appears to be phospho-ubiquitination (Figure 1). A corresponding signal in yeast cells has not been definitely identified at this point, although it is very likely to involve post-translational modifications of Atg32, as discussed above. Indeed, recent studies have indicated that Atg32 is proteolytically processed at its C-terminus and that this step is essential for mitophagy [39].

Several lines of investigation suggest that mitochondrial components are sorted prior to mitophagy, and that the degraded components are not a random representation of mitochondrial biomass. The observation that mitochondrial fission results in one hyperpolarized and one depolarized daughter [13], together with the observation that mitochondrial content mixing, not mitochondrial size, is the determinant for the rate of mitophagy, both suggest a distillation-type mechanism for separating intra-mitochondrial components [43]. Indeed, a SILAC (stable isotope labelling by amino acids in cell culture)-based proteomic screen identified mitochondrial matrix proteins with widely diverging rates of mitophagy [44]. Strikingly, mitochondrial matrix proteins which are inefficiently degraded by mitophagy are clearly segregated from those undergoing efficient mitophagy, within the matrix: inefficiently degraded proteins seem to be concentrated in specific intra-mitochondrial foci, whereas those undergoing efficient mitophagy are evenly dispersed in the mitochondrial matrix [44]. One possibility is that this reflects a distillation process that is coupled to mitochondrial dynamics on the one hand, and mitophagic engulfment on the other [45]. Interestingly, Mao et al reported a direct interaction between Atg11, an autophagy factor that functions in cargo selectivity, and Dnm1, a dynamin like FTPase required for mitochondrial fission [46]. Mitochondrial fission is known to occur at ER-mitochondrial contact sites [47], and ER contact sites were shown to be important for induction of mitophagy [48].

In mammals, it was found that upon differentiation of induced pluripotent stem cells (iPSCs) derived from patients carrying a mitochondrial tRNA mutation to neuronal lineages (MELAS-iPSC), mitochondrial electron transport complex I undergoes selective mitophagy that appears to involve intra-mitochondrial protein segregation [49,50]. In addition it was found that the mutant DNA also undergoes a segregation effect, as differentiation involved a shift to homoplasmy that did not involve any reduction in mtDNA copy number [49]. Additional support for the concept of intra-mitochondrial selectivity during mitophagy comes from SILAC studies using mice [51]. These found that the half-lives of proteins from heart and skeletal muscle mitochondria in these tissues could vary from hours to months, a difference that is incompatible with non-selective mitophagy.

\section{Concluding remarks}

Recent work in mammalian cells has made great strides in our understanding of mitophagy. On the other hand, the discovery of stationary phase mitophagy in respiring yeast cells has opened the door to high-throughput genomic screens, and has allowed the identification of factors required for this process. Studies in both systems have indicated that distinct signalling mechanisms identify mitochondria that are destined for mitophagy. However we are beginning to appreciate that these signalling mechanisms must also be coupled to intra-mitochondrial mechanisms that sort defective, perhaps oxidized, factors. It is possible that these signals activate and use the mitochondrial fission and fusion machinery to 'distill' defective components out of the general mitochondrial milieu. One therefore can also postulate at least two signalling events, not one: The original overall physiological burden as sensed by the cells due to accumulation of unsorted mitochondrial damage may induce the 'sorting' process, whereas the segregation of defective mitochondrial compartments could induce a specific 'eat me' signal mediated by phospho-ubiquitin in mammals and by Atg32 modifications in yeast. Within this hypothetical framework, different classes of genes, having different effects on the various stages described, would be predicted to contribute to the overall process. Much more work, both scientific and theoretical, will be needed in order to test these hypotheses and to identify the responsible molecular machinery in the different mitochondrial compartments.

\section{Funding}

This work is supported by the Israel Science Foundation [grant number 422/12]; the German-Israel Research Foundation [grant number 1297]; the Freiburg Institute for Advanced Studies (to H.A. and J.D.); and we also acknowledge funding from the People Program (Marie Curie Actions) of the European Union's Seventh Framework Programme (FP7/2001-2013) under REA [grant agreement number 609305].

\section{References}

1 Balaban, R.S., Nemoto, S. and Finkel, T. (2005) Mitochondria, oxidants, and aging. Cell 120, 483-495 CrossRef PubMed

2 Wallace, D.C. (2005) A mitochondrial paradigm of metabolic and degenerative diseases, aging, and cancer: a dawn for evolutionary medicine. Annu. Rev. Genet. 39, 359-407 CrossRef PubMed

3 Wallace, D.C. (1999) Mitochondrial diseases in man and mouse. Science 283, 1482-1488 CrossRef PubMed

4 Ahlqvist, K.J., Suomalainen, A. and Hämäläinen, R.H. (2015) Stem cells, mitochondria and aging. Biochim. Biophys. Acta 1847, 1380-1386 CrossRef PubMed

5 HARMAN, D. (1956) Aging: a theory based on free radical and radiation chemistry. J. Gerontol. 11, 298-300 CrossRef PubMed

6 Wang, T. and Hay, J.C. (2015) Alpha-synuclein toxicity in the early secretory pathway: how it drives neurodegeneration in Parkinsons disease. Front. Neurosci. 9, 433 PubMed

7 Greenamyre, J.T., MacKenzie, G., Peng, T.I. and Stephans, S.E. (1999) Mitochondrial dysfunction in Parkinson's disease. Biochem. Soc. Symp. 66, 85-97 CrossRef PubMed

8 Kumaran, R. and Cookson, M.R. (2015) Pathways to Parkinsonism Redux: convergent pathobiological mechanisms in genetics of Parkinson's disease. Hum. Mol. Genet. 24, R32-R44 CrossRef PubMed

9 Rodriguez-Enriquez, S., He, L. and Lemasters, J.J. (2004) Role of mitochondrial permeability transition pores in mitochondrial autophagy. Int. J. Biochem. Cell Biol. 36, 2463-2472 CrossRef PubMed 
10 Rodriguez-Enriquez, S., Kai, Y., Maldonado, E., Currin, R.T. and Lemasters, J.J. (2009) Roles of mitophagy and the mitochondrial permeability transition in remodeling of cultured rat hepatocytes. Autophagy $\mathbf{5}$, 1099-1106 CrossRef Pubmed

11 Rodriguez-Enriquez, S., Kim, I., Currin, R.T. and Lemasters, J.J. (2006) Tracker dyes to probe mitochondrial autophagy (mitophagy) in rat hepatocytes. Autophagy 2, 39-46 CrossRef PubMed

12 Narendra, D., Tanaka, A., Suen, D.F. and Youle, R.J. (2008) Parkin is recruited selectively to impaired mitochondria and promotes their autophagy. J. Cell Biol. 183, 795-803 CrossRef PubMed

13 Twig, G., Elorza, A., Molina, A.J., Mohamed, H., Wikstrom, J.D., Walzer, G., Stiles, L., Haigh, S.E., Katz, S., Las, G. et al. (2008) Fission and selective fusion govern mitochondrial segregation and elimination by autophagy. EMBO j 27, 433-446 CrossRef PubMed

14 Jin, S.M. Lazarou, M. Wang, C., Kane, L.A., Narendra, D.P. and Youle, R.t. (2010) Mitochondrial membrane potential regulates PINK1 import and proteolytic destabilization by PARL. J. Cell Biol. 191, 933-942 CrossRef PubMed

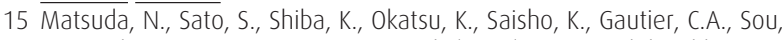
Y.S., Saiki, S., Kawajiri, S., Sato, F. et al. (2010) PINK1 stabilized by mitochondrial depolarization recruits Parkin to damaged mitochondria and activates latent Parkin for mitophagy. J. Cell Biol. 189, 211-221 CrossRef PubMed

16 Narendra, D.P., Jin, S.M., Tanaka, A., Suen, D.F., Gautier, C.A., Shen, J., Cookson, M.R. and Youle, R.J. (2010) PINK1 is selectively stabilized on impaired mitochondria to activate Parkin. PLoS Biol 8, e1000298 CrossRef PubMed

17 Kondapalli, C., Kazlauskaite, A., Zhang, N., Woodroof, H.I., Campbell, D.G., Gourlay, R., Burchell, L., Walden, H., Macartney, T.J., Deak, M. et al. (2012) PINK1 is activated by mitochondrial membrane potential depolarization and stimulates Parkin E3 ligase activity by phosphorylating Serine 65. Open Biol 2, 120080 CrossRef PubMed

18 Koyano, F., Okatsu, K., Kosako, H., Tamura, Y., Go, E., Kimura, M., Kimura, Y., Tsuchiya, H., Yoshihara, H., Hirokawa, T. et al. (2014) Ubiquitin is phosphorylated by PINK1 to activate parkin. Nature 510, 162-166 pubmed

19 Shiba-Fukushima, K., Imai, Y., Yoshida, S., Ishihama, Y., Kanao, T., Sato, S. and Hattori, N. (2012) PINK1-mediated phosphorylation of the Parkin ubiquitin-like domain primes mitochondrial translocation of Parkin and regulates mitophagy. Sci. Rep. 2, 1002 CrossRef PubMed

20 Kazlauskaite, A., Kondapalli, C., Gourlay, R., Campbell, D.G., Ritorto, M.S. Hofmann, K., Alessi, D.R., Knebel, A., Trost, M. and Muqit, M.M. (2014) Parkin is activated by PINK1-dependent phosphorylation of ubiquitin at Ser65. Biochem. J. 460, 127-139 CrossRef PubMed

21 Kane, L.A., Lazarou, M., Fogel, A.I., Li, Y., Yamano, K., Sarraf, S.A., Banerjee, S. and Youle, R.J. (2014) PINK1 phosphorylates ubiquitin to activate Parkin E3 ubiquitin ligase activity. J. Cell Biol. 205, 143-153 CrossRef Pubmed

22 Ordureau, A., Sarraf, S.A., Duda, D.M., Heo, J.M., Jedrychowski, M.P. Sviderskiy, V.O., Olszewski, J.L., Koerber, J.T., Xie, T., Beausoleil, S.A. et al. (2014) Quantitative proteomics reveal a feedforward mechanism for mitochondrial PARKIN translocation and ubiquitin chain synthesis. Mol. Cell 56, 360-375 CrossRef PubMed

$23 \mathrm{Heo}$, J.M., Ordureau, A., Paulo, J.A., Rinehart, J. and Harper, J.W. (2015) The PINK1-PARKIN mitochondrial ubiquitylation pathway drives a program of OPTN/NDP52 recruitment and TBK1 activation to promote mitophagy. Mol. Cell 60, 7-20 CrossRef PubMed

24 Lazarou, M., Sliter, D.A., Kane, L.A., Sarraf, S.A., Wang, C., Burman, J.L., Sideris, D.P., Fogel, A.I. and Youle, R.J. (2015) The ubiquitin kinase PINK1 recruits autophagy receptors to induce mitophagy. Nature 524, 309-314 CrossRef Pubmed

25 Wild, P., Farhan, H., McEwan, D.G., Wagner, S., Rogov, V.V., Brady, N.R., Richter, B., Korac, J., Waidmann, 0., Choudhary, C. et al. (2011) Phosphorylation of the autophagy receptor optineurin restricts Salmonella growth. Science 333, 228-233 CrossRef PubMed

26 Thurston, T.L., Ryzhakov, G., Bloor, S., von Muhlinen, N. and Randow, F. (2009) The TBK1 adaptor and autophagy receptor NDP52 restricts the proliferation of ubiquitin-coated bacteria. Nat. Immunol 10, 1215-1221 CrossRef PubMed

27 Chen, Y. and Dorn, G.W. (2013) PINK1-phosphorylated mitofusin 2 is a Parkin receptor for culling damaged mitochondria. Science $\mathbf{3 4 0}$ 471-475 PubMed

28 Tanaka, A., Cleland, M.M., Xu, S., Narendra, D.P., Suen, D.F., Karbowski, M. and Youle, R.J. (2010) Proteasome and p97 mediate mitophagy and degradation of mitofusins induced by Parkin. J. Cell Biol. 191, 1367-1380 crossRef PubMed
29 Takeshige, K., Baba, M., Tsuboi, S., Noda, T. and Ohsumi, Y. (1992) Autophagy in yeast demonstrated with proteinase-deficient mutants and conditions for its induction. J. Cell Biol. 119, 301-311 CrossRef PubMed

30 Deter, R.L., Baudhuin, P. and De Duve, C. (1967) Participation of lysosomes in cellular autophagy induced in rat liver by glucagon. J. Cell Biol. 35, C11-C16 CrossRef PubMed

31 Kissova, I., Deffieu, M., Manon, S. and Camougrand, N. (2004) Uth1p is involved in the autophagic degradation of mitochondria. J. Biol. Chem. 279, 39068-39074 CrossRef PubMed

32 Tal, R., Winter, G., Ecker, N., Klionsky, D.J. and Abeliovich, H. (2007) Aup1p, a yeast mitochondrial protein phosphatase homolog, is required for efficient stationary phase mitophagy and cell survival. J. Biol. Chem. 282, 5617-5624 CrossRef PubMed

33 Journo, D., Mor, A. and Abeliovich, H. (2009) Aup1-mediated regulation of Rtg3 during mitophagy. J. Biol. Chem. 284, 35885-35895 CrossRef Pubmed

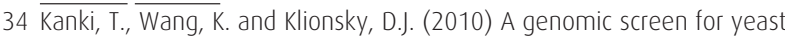
mutants defective in mitophagy. Autophagy 6, 278-280

35 Kanki, T., Wang, K., Cao, Y., Baba, M. and Klionsky, D.J. (2009) Atg32 is a mitochondrial protein that confers selectivity during mitophagy. Dev. Cell 17, 98-109 CrossRef Pubmed

36 Okamoto, K., Kondo-Okamoto, N. and Ohsumi, Y. (2009) Mitochondria-anchored receptor Atg32 mediates degradation of mitochondria via selective autophagy. Dev. Cell 17, 87-97 CrossRef PubMed

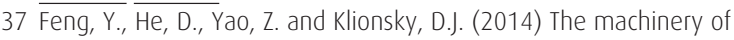
macroautophagy. Cell Res 24, 24-41 CrossRef PubMed

38 Aoki, Y., Kanki, T., Hirota, Y., Kurihara, Y., Saigusa, T., Uchiumi, T. and Kang, D. (2011) Phosphorylation of Ser114 on Atg32 mediates mitophagy. Mol. Biol. Cell 22, 3206-3217

39 Wang, K., Jin, M., Liu, X. and Klionsky, D.J. (2013) Proteolytic processing of Atg32 by the mitochondrial i-AAA protease Yme1 regulates mitophagy. Autophagy 9, 1828-1836 CrossRef PubMed

40 Sakakibara, K., Eiyama, A., Suzuki, S.W., Sakoh-Nakatogawa, M. Okumura, N., Tani, M., Hashimoto, A., Nagumo, S., Kondo-Okamoto, N., Kondo-Kakuta, C. et al. (2015) Phospholipid methylation controls Atg32-mediated mitophagy and Atg8 recycling. EMBO J 34, 2703-2719 CrossRef PubMed

41 Ziviani, E., Tao, R.N. and Whitworth, A.J. (2010) Drosophila parkin requires PINK1 for mitochondrial translocation and ubiquitinates mitofusin. Proc. Natl. Acad. Sci. U.S.A. 107, 5018-5023 CrossRef PubMed

42 Ziviani, E. and Whitworth, A.). (2010) How could Parkin-mediated ubiquitination of mitofusin promote mitophagy? Autophagy 6, 660-662 CrossRef PubMed

43 Abeliovich, H. (2011) Stationary-phase mitophagy in respiring Saccharomyces cerevisiae. Antioxid. Redox Signal 14, 2003-2011 CrossRef Pubmed

44 Abeliovich, H., Zarei, M., Rigbolt, K.T., Youle, R.J. and Dengjel, J. (2013) Involvement of mitochondrial dynamics in the segregation of mitochondrial matrix proteins during stationary phase mitophagy. Nat. Commun 4, 2789 CrossRef PubMed

45 Dengjel, J. and Abeliovich, H. (2014) Musical chairs during mitophagy. Autophagy 10, 706-707 CrossRef PubMed

46 Mao, K., Wang, K., Liu, X. and Klionsky, D.J. (2013) The scaffold protein Atg11 recruits fission machinery to drive selective mitochondria degradation by autophagy. Dev. Cell 26, 9-18 CrossRef PubMed

47 Friedman, J.R., Lackner, L.L., West, M., DiBenedetto, J.R., Nunnari, J. and Voeltz, G.K. (2011) ER tubules mark sites of mitochondrial division. Science 334, 358-362 CrossRef PubMed

48 Böckler, S. and Westermann, B. (2014) Mitochondrial ER contacts are crucial for mitophagy in yeast. Dev. Cell 28, 450-458

49 Hämäläinen, R.H., Manninen, T., Koivumäki, H., Kislin, M., Otonkoski, T. and Suomalainen, A. (2013) Tissue- and cell-type-specific manifestations of heteroplasmic mtDNA 3243A>G mutation in human induced pluripotent stem cell-derived disease model. Proc. Natl. Acad. Sci. U.S.A. 110, E3622-E3630 CrossRef PubMed

50 Pickrell, A.M. and Youle, R.J. (2013) Mitochondrial disease: mtDNA and protein segregation mysteries in iPSCs. Curr. Biol. 23, R1052-R1054 CrossRef PubMed

51 Kim, T.Y., Wang, D., Kim, A.K., Lau, E., Lin, A.J., Liem, D.A., Zhang, J., Zong, N.C., Lam, M.P. and Ping, P. (2012) Metabolic labeling reveals proteome dynamics of mouse mitochondria. Mol. Cell Proteomics 11, 1586-1594 CrossRef PubMed 